\title{
Tumor Detection Using Trapezoidal Function and Rayleigh Distribution.
}

\author{
"Mohammad Aslam. C ${ }^{1}$ Satya Narayana. D 2 Padma Priya. $\mathrm{K}^{3}$ \\ ${ }^{I}$ Vaagdevi Institute of Technology and Science, Proddatur, A.P \\ ${ }^{2}$ Rajiv Gandhi Memorial College Of Engineering And Technology, Nandyal, A.P \\ ${ }^{3}$ jntuk College of Engineering, Kakinada, A.P \\ Corresponding Author: Mohammad Aslam. $C^{l}$
}

\begin{abstract}
Breast cancer is one of the most common cancers worldwide. In developed countries, among one in eight women develop breast cancer at some stage of their life. Early diagnosis of breast cancer plays a very important role in treatment of the disease. With the goal of identifying genes that are more correlated with the prognosis of breast cancer. An algorithm based on Trapezoidal and Rayleigh distributions. Property is used for the detection of the cancer tumors. Fuzzy logic and markov random fields work in real time. We also try to show its performance by comparing it with the existing real time algorithm that uses only markov random fields. This is not only suitable for the detection of the tumors, but also suitable for various general applications like in the detection of object in an image etc.
\end{abstract}

Keywords: Tumors, Rayleigh distribution texture, Segmentation, Fuzzy Logic.

\section{Introduction}

Breast Cancer is one of the main sources of malignancy related passings in ladies. There are accessible distinctive strategies for the discovery of malignancy tumors, these techniques are utilized for the finding of disease in ladies at early stages. This can be a troublesome assignment because of the unpredictable size and state of the tumor. One of the techniques utilized for tumor discovery depends on Computed Tomography picture. In this technique we utilize the probabilistic pixel choice approach. The division procedure here is performed in 2 dimensional cut-to-cut premises. The divided cuts are put away as an informational collection. In this strategy we utilize diverse seed focuses for various test sets. This technique turned out to be effective for a few information sets where concerning a few informational indexes it has a few detriments. The precision of the tumor identification in view of the dataset we will test. Modernized picture based location strategy can likewise be utilized for the tumor location. In this strategy we go for Lymphocytic Infiltration. This LI capacity as a potential against tumor component. In this strategy pathologists and oncologists go for manual location of tumors. Here quantitative components are gotten from charts utilizing the techniques like Voronoi Diagram, Delaunay triangulation and least traversing tree. The assessment strategies that are utilized here are Hausdorff Distance; Cross Validation utilizing SVM classifier, VZ Texton based classifier. This technique is being mechanized by utilizing CADx framework. Here we utilize locale developing calculations and consequent MRF based refinement to confine LI from the encompassing BC cores, pattern level of Lymphocytes. Division of the tumor can likewise be performed utilizing Gaussian Markov Random Fields. In this technique the whole picture is accepted such it is having just two classes and it is demonstrated utilizing Rayleigh dissemination. The encoding for spatial communications between pixels, its parameters can be effectively assessed utilizing Least squares. There strategies are appropriate for continuous applications. The division performed here is regulated division, in which it is partitioned into two stages. In the first place stage is highlight choice and second stage is trying the chosen highlights utilizing the division calculation. The recognition precision of this strategy is great contrasted with different strategies that are accessible. The mistake rate is likewise less for this technique.

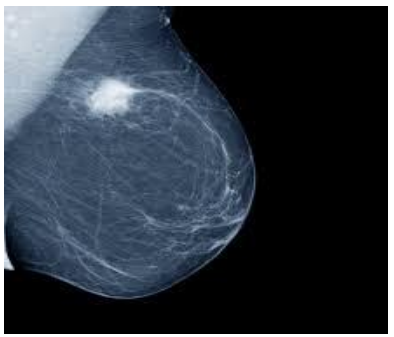

Fig 1: Typical picture of tumor 
In this paper for the recognition of tumor we utilize a calculation created by utilizing straightforward Trapezoidal and Rayleigh conveyances.. The point of this review is to build up a vigorous calculation for discovery of tumors. We utilize an ongoing division calculation for this. Markov Random Fields encourages us to manage the instabilities in a picture by methods for expressly characterizing the spatial collaborations between pixels as a likelihood appropriation. The picture is expected with the end goal that it is having just 2 classes and it is demonstrated utilizing Rayleigh conveyance. Rayleigh circulation is one of the most straightforward MRF that is reasonable for encoding spatial connections amongst pixels and can be effectively assessed utilizing slightest squares. The expression "Fluffy Logic" was presented by Latfi A. Zadeh. Fluffy rationale has been connected to many fields, from control hypothesis to counterfeit consciousness. This Fuzzy rationale utilizes edge discovery strategy to discover the tumor edge pixels by applying the standards to every pixel alongside its 8-neighborhood pixels. Before applying the tenets, every pixel is classified into a "white" and "dark" pixel utilizing the triangular participation work.

Whatever remains of paper is sorted out as takes after. Segment 2 gives essential definitions and documentations of MRF hypothesis. Organization 3 portrays about Rayleigh conveyance. The fluffy rationale with edge identification is depicted in Sects.4. The Image model is spoken to in organization 5. The execution examination for existing and proposed strategies is talked about in Sect.6 taken after with conclusion.

\section{Rayleigh Distribution Function}

A Rayleigh distribution is often observed when the overall magnitude of a vector is related to its directional components. One example where the Rayleigh distribution naturally arises is when wind velocity is analyzed into its orthogonal 2-dimensional vector components. Assuming that each component is uncorrelated, normally distributed with equal variance, and zero mean, then the overall wind speed (vector magnitude) will be characterized by a Rayleigh distribution. A second example of the distribution arises in the case of random complex numbers whose real and imaginary components are independently and identically distributed. Gaussian with equal variance and zero mean. In that case, the absolute value of the complex number is Rayleighdistributed. The distribution is named after Lord Rayleigh. The probability density function of the Rayleigh distribution

$$
\left.\mathbf{f}(\mathbf{x} ; \boldsymbol{\sigma})=\frac{\mathbf{x}}{\boldsymbol{\sigma}^{2}} \mathrm{e}^{-\frac{\mathrm{x}^{2}}{(2}} \boldsymbol{\sigma}^{2}\right), \quad \mathbf{x} \geq \mathbf{0}
$$

Where is the scale parameter of the distribution. The cumulative distribution is

$$
\left.F(x)=1-e^{-\frac{x^{2}}{(2}} \sigma^{2}\right) \text { for } x \in[0, \infty)
$$

Consider the 2-D vector $Y=(U ; V)$ which has components that are Gaussian-distributed, centered at zero, and independent.

By transforming to the polar coordinate system then

$$
f(x, \sigma)=\frac{1}{2 \pi \sigma^{2}} \int_{-\infty}^{\infty} d u \int_{-\infty}^{\infty} d v e^{\frac{-u^{2}}{2 \sigma^{2}}} e^{\frac{-v^{2}}{2 \sigma^{2}}} \delta\left(x-\sqrt{u^{2}+v^{2}}\right)
$$

$$
\mathbf{f}(\mathbf{x}, \sigma)=\frac{1}{2 \pi \sigma^{2}} \int_{-\infty}^{\infty} \mathrm{d} \emptyset \int_{0}^{\infty} \mathrm{dr} \quad \mathbf{e}^{\frac{-\mathbf{r}^{2}}{2 \sigma^{2}}} \delta(\mathbf{r}-\mathbf{x}) \mathbf{r}=\frac{x}{\sigma^{2}} e^{\frac{-x^{2}}{2 \sigma^{2}}}
$$

which is the Rayleigh distribution. It is straightforward to generalize to vectors of dimension other than 2 . There are also generalizations when the components have unequal variance or correlations.

\section{Applications}

An application of the estimation of ' $\sigma$ ' can be found in magnetic resonance imaging (MRI). As MRI images are recorded as complex images but most often viewed as magnitude images, the background data is Rayleigh distributed. Hence, the above formula can be used to estimate the noise variance in an MRI image from background data.

\subsection{Definition of Fuzzy Logic:}

\section{Fuzzy Logic}

Fuzzy logic is a mathematical logic that attempts to solve problems by assigning values to an imprecise spectrum of data in order to arrive at the most accurate conclusion possible. Here, the fuzzy logic is used to conclude whether a pixel is an edge pixel or not. The proposed technique begins by fuzzyfying the gray values of a pixel into two fuzzy variables, namely the black and the white. Fuzzy rules are defined to find the edge pixels in the fuzzified images. The term "fuzzy logic" was introduced with the proposal of fuzzy set theory by Lotfi A. zadeh. Fuzzy logic has been applied to many fields, from control theory to artificial intelligence. This Fuzzy logic uses Edge detection method to find the weed edge pixels, by applying the rules to each pixel along with its 8-neighbourhood. Before applying the rules, each pixel is categorized into a "white" and "black" pixel using the triangular membership function. 
Tumor Detection Using Trapezoidal and Rayleigh Distribution.

\subsection{Fuzzy with Edge detection:}

Edge detection plays a vital role in many of the applications of image processing such as pattern recognition and image segmentation. Edges correspond to sharp variations of image intensity and convey vital information in an image. Edges are formed from pixels with derivative values that exceed a pre-set threshold. Edge detection not only extracts the edges of the interested objects from an image, but it also forms the basis for image fusion, shape extraction, image matching, image tracking Normally, the edge detection methods use the gradient of images and arithmetic operators. The most popular edge detection methods, such as Sobel, Prewitt, Roberts etc. detect edges using a first-order derivative of intensity since they consider edges to be a set of pixels where there is an abrupt change in the intensity of the gray level. These edge detection methods do not consider the neighborhood of a pixel, while in our proposed method plays an important role in edge detection.On the other hand, fuzzy logic employs simple if-then rules, which do not require any thresholding or complex gradient based calculations. Thus, an edge detection method that is based on fuzzy logic is proposed in our project.

Here are a lot of ways to detect edges using fuzzy image processing. But the simplest way is to fuzzify the image. This involves finding the membership value of each pixel for a particular set and then applying the defined rules to the fuzzified image to find the edge map. If the information in a database is inexact, incomplete, or not entirely certain, then the systematic use of fuzzy logic becomes practically indispensable. In many image processing applications, the image information that is to be processed is uncertain and imprecise. In the proposed approach, the question of whether a pixel is darker or brighter comes under the realm of fuzzification. The darker pixels are placed in the black class, whereas, the brighter ones are put in the white class. In order to fuzzify the image, the membership of each pixel is found by using the triangular membership function. The membership function of an element defines the degree to which that element belongs to the fuzzy set. The value of the membership function always lies between $[0 \ldots 1]$.

\subsection{Fuzzy Rules:}

People settle on choices in view of principles. The choice and the methods for picking that choice are supplanted by fluffy sets and the standards are supplanted by fluffy tenets. A fluffy run is characterized as a contingent explanation as:

$$
\text { If } \mathrm{x}=\mathrm{A} \text { then }
$$

At that point $\mathrm{y}=\mathrm{B}$

Where $\mathrm{x}$ and $\mathrm{y}$ are phonetic factors, $\mathrm{A}$ and $\mathrm{B}$ are semantic esteems that are controlled by fluffy sets on the universe of the talk $\mathrm{X}$ and $\mathrm{Y}$, separately. The fluffy principles utilized as a part of the proposed edge location approach consider the phonetic estimations of the 8-neighborhood of the pixel that is under thought. Here, the etymological esteems can be white or dark. The fluffy run is a control framework that is utilized to derive choices from a learning base. The learning base to construe the edge pixel in a picture is the pixel with its 8-neighborhood. The choice whether every pixel is an edge pixel or not is made by utilizing the fluffy standards that are connected to the 8-neighborhood. The pixels in the 8-neighborhood of a pixel may be dark or white.

\section{Trapezoidal Function}

The trapezoidal function is defined by minimum, lower mode, upper mode, and maximum parameters. The generalized trapezoidal distribution adds three more parameters: the growth rate, decay rate, and boundary ratio parameters. Van Dorp and Kotz[2] and van Dorp and colleagues[1] formally describe the generalized trapezoidal distribution, representing the minimum, lower mode, upper mode, maximum, growth rate, decay rate, and boundary ratio with parameters $\mathrm{a} ; \mathrm{b} ; \mathrm{c} ; \mathrm{d} ; \mathrm{m} ; \mathrm{n} ;$ and, respectively. The probability density function of the generalized trapezoidal distribution with parameters $\mathrm{a} ; \mathrm{b} ; \mathrm{c} ; \mathrm{d} ; \mathrm{m} ; \mathrm{n}$; and _ is given by:

$$
\mu(\mathrm{x}, \mathrm{a}, \mathrm{b}, \mathrm{c}, \mathrm{d})=\left\{\begin{array}{lc}
\frac{x-a}{b-a}, & \text { if } a \leq x \leq b \\
1, & \text { if } b<x<c \\
\frac{d-x}{d-c}, & \text { if } c \leq x<d \\
0, & \text { if } d<x
\end{array}\right.
$$

Where $\mathrm{x}=$ variable,

$\mathrm{a}, \mathrm{b}, \mathrm{c}, \mathrm{d}$ are parameters.

The trapezoid package provides functions for the probability density function (d trapezoid), cumulative distribution function ( $\mathrm{p}$ trapezoid), quantile function ( $\mathrm{q}$ trapezoid), and random generation ( $\mathrm{r}$ trapezoid). The 
parameters a; b; c; d; m; n; and _ are specified by the arguments min, mode1, mode2, max, n1, n3, and alpha, respectively. The argument names were chose into avoid conflicts with names that commonly have specific meaning in $\mathrm{R}$ functions such as $\mathrm{C}$ and $\mathrm{n}$.

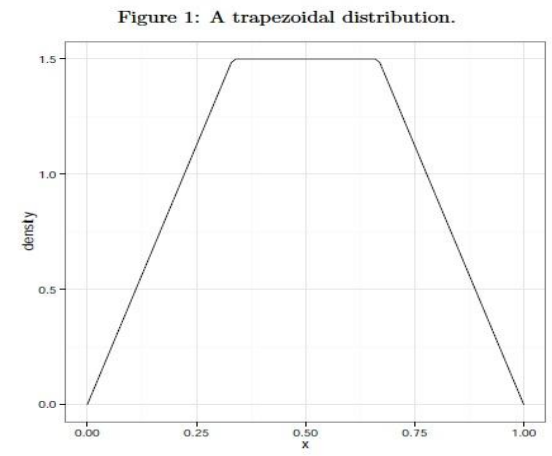

Fig 1: Trapizoidal function

\section{Image Model}

Tumor can be detected by using the Rayleigh distribution but, this approach cannot detect the noisy images and so to overcome this drawback, we developed here a new algorithm that uses edge detection with fuzzy logic along with Rayleigh distribution property. In this newly developed algorithm first we take an RGB color image of size $\mathrm{M} \times \mathrm{N}$ and convert it to grayscale image. Then apply the fuzzy logic. For this we need to take histogram of the grayscale image. Based on this we have to select the fuzzy variables 'a' and 'c' manually. Now apply the fuzzy rules. Then convert the matrix image into grayscale image. Now Rayleigh distribution property is to be applied. For this every time take two $3 \times 3$ matrices and find the absolute difference between the pixels of two matrices. If it is equals to 1 then compute s. Otherwise compute s1. Combining s and s1 calculate new matrix. Then convert this into grayscale image and finally we get an output image which consists of tumor only. This approach can even detect the images that are corrupted by noise.

\section{Performance Measurement}

To compare the performance of the proposed method with the existing method, here we add noise for both existing method and the proposed method.

\section{Salt \& Pepper noise.}

After adding these noise we compare the original image with the noise added image. Now draw the graph for noise by noting their values. This process is same for both existing and proposed methods. Their results are as shown in below figures.

TABLE 1: Comparison of outputs of Rayleigh distribution and Fuzzy Logic Method with Different Threshold Value.

\begin{tabular}{|c|c|c|c|}
\hline Original Image & $\begin{array}{c}\text { GMRF } \\
\text { Output }\end{array}$ & $\begin{array}{c}\text { Rayleigh distribution with } \\
\text { Fuzzy Logic Output }\end{array}$ & $\begin{array}{c}\text { Threshold Value for Fuzzy Logic } \\
(\mathrm{a}, \mathrm{c})\end{array}$ \\
\hline & & 7 & 60,250 \\
\hline & & & \\
\hline
\end{tabular}


Tumor Detection Using Trapezoidal and Rayleigh Distribution.

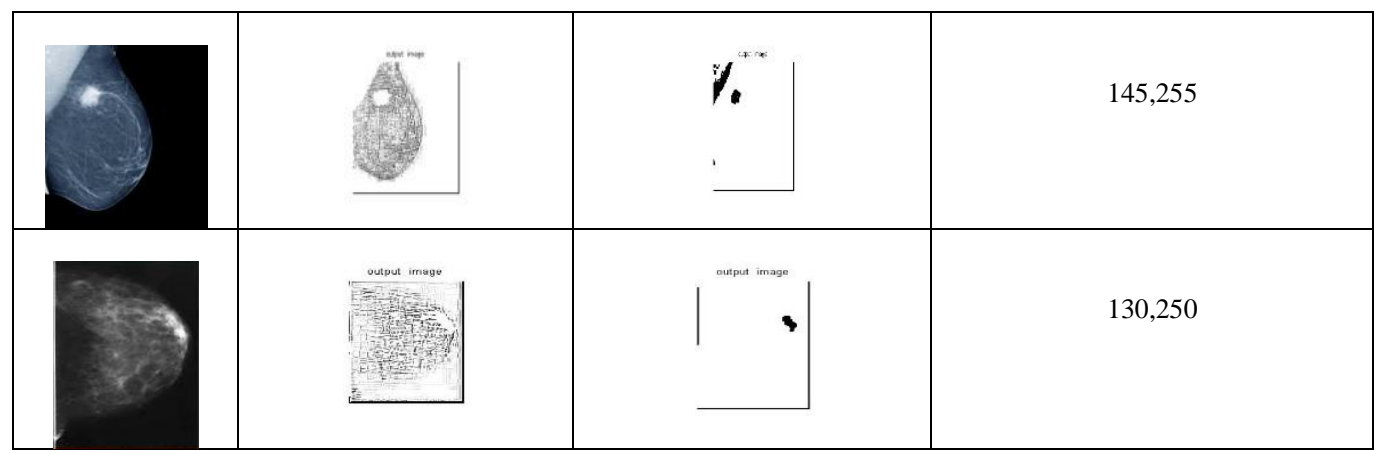

TABLE2: Comparison of outputs of GMRF and Rayleigh distribution with Fuzzy Logic Method with Salt \& Pepper Noise.

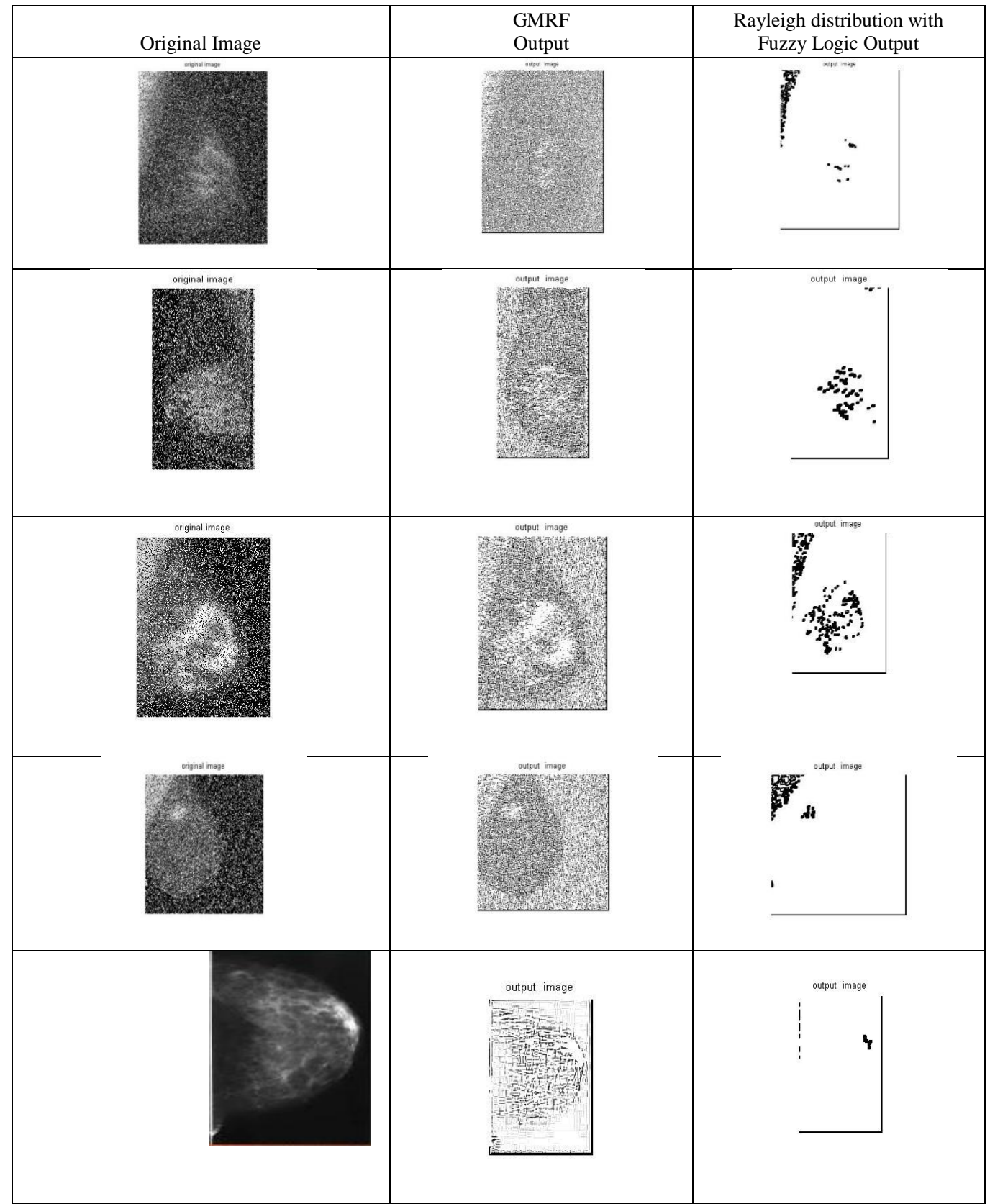

VIII. Conclusion

This venture can effectively distinguish the tumor which can be utilized as a part of therapeutic analysis. So through this venture we can obviously demonstrate that our proposed strategy will give acceptable 
outcomes contrasted with the current technique. And furthermore this is reasonable for location of pictures that are debased by commotions, Moreover this venture is appropriate for different broadly useful applications as said beforehand too. The future extent of this work is programmed determination of the limit esteems.

\section{References}

[1] Mohammad Aslam. C, Dr. Satya Narayana. D, Dr. Padma Priya. K, Segmentation of RumexObtusifolius using Simple Trapezoidal and Rayleigh distributions., Unpublished.

[2] Ajay NageshBasavanhally, ShridarGanesan, Shannon Agner, James Peter Monaco, Michael D. Feldman, John E. Tomaszewski, GyanBhanot and AnantMadabhushi, Computerized Image-Based Detection and Grading of Lymphocytic Infiltration in HER2+ Breast Cancer Histopathology, IEEE Transactions on Biomedical Engineering, vol. 57, no. 3, pp.642-653, march 2010.

[3] O.P.Verma, Vein Jain and RajiniGumber, Simple Fuzzy Rule Based Edge Detection, J Inf Process Syst, Vol.9, No.4, pp.575-591, December 2013.

[4] Santosh Hiremath, Valentyn A. Tolpekin, Gerie van der Heijden, Alfred Stein, Segmentation of Rumex obtusifoliususing Gaussian Markov random fields, Machine Vision and Applications (2013) 24:845-854, Springer.

[5] Jung LengFoo, GoMiyano, ThomLobe, EliotWiner,Tumor segmentation from computed tomography image data using a probabilistic pixel selection approach, Computers in Biology and Medicine 41 (2011) 56-65, ELSEVIER.

[6] R.Radha, P.Rajendiran,Using K-Means Clustering Technique To Study Of Breast Cancer, 2014 World Congress on Computing and Communication Technologies (211-214).

[7] H. Abdellatif, T. E. Taha, O. F. Zahran, W. Al-Nauimy, F. E. Abd El-Samie,Automatic Segmentation of Digital Mammograms to Detect Masses, NRSC 2013 (557-565).

[8] P.G.K.Sirisha, R. Pradeep Kumar Reddy, C. Naga Raju “ An Efficient Fuzzy Technique for Detection of Brain Tumor”, International journal of computers \& technology(IJCT) Vol 8, No 2,2013.

[9] C. Naga Raju, HariKiran. C, Siva Priya. T "Design of Primary Screening Software Testing Tool for Early Detection of Breast Cancer", Journal of Advances in Information Technology (JAIT, ISSN 1798-2340), 2012.

IOSR Journal of Electronics and Communication Engineering (IOSR-JECE) is UGC approved Journal with Sl. No. 5016, Journal no. 49082. 